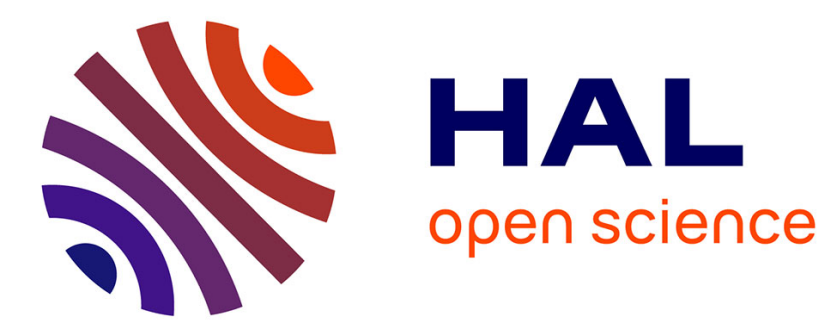

\title{
Performance Modeling of SEAD Protocol in Vehicular Environment
}

\author{
Imen Achour, Tarek Bejaoui, Anthony Busson, Sami Tabbane
}

\section{To cite this version:}

Imen Achour, Tarek Bejaoui, Anthony Busson, Sami Tabbane. Performance Modeling of SEAD Protocol in Vehicular Environment. Wireless Networks, 2018, pp.1-18. 10.1007/s11276-018-1706-3 . hal-01782477

\section{HAL Id: hal-01782477 \\ https://hal.inria.fr/hal-01782477}

Submitted on 2 May 2018

HAL is a multi-disciplinary open access archive for the deposit and dissemination of scientific research documents, whether they are published or not. The documents may come from teaching and research institutions in France or abroad, or from public or private research centers.
L'archive ouverte pluridisciplinaire HAL, est destinée au dépôt et à la diffusion de documents scientifiques de niveau recherche, publiés ou non, émanant des établissements d'enseignement et de recherche français ou étrangers, des laboratoires publics ou privés. 


\title{
Performance Modeling of SEAD Protocol in Vehicular Environment
}

\author{
Imen Achour \\ Tarek Bejaoui \\ Anthony Busson \\ Sami Tabbane
}

May 2, 2018

\begin{abstract}
Toward the growing number of the transport safety applications offered by VANET, a large amount of messages need to be efficiently disseminated between connected vehicles. The requirement for an efficient data dissemination in vehicular environment is ensuring the highest data reachability, using the minimum network resources within a short end to end delay. To meet these properties, researchers have addressed the data dissemination challenges, mainly the famous broadcast storm, which has lead to a wide variety of solutions. In this work, we present two Simple and Efficient Adaptive data Dissemination protocols called SEAD1 and SEAD2 that combine a probabilistic and a delay based approach. The originality of these protocols is that they can be adaptively tuned according to the VANET applications' requirements. Therefore, a rigorous performance evaluation of the protocols is proposed through an analytic model and a large set of NS3 simulations. The analytic results show excellent fits to the simulation results which confirm the accuracy of the proposed model. Moreover, based on our analytic model we became able to thoroughly study the protocols parameters' behavior and derive an accurate configuration. Thereby, a proper trade-off between the packet delivery ratio and the redundancy ratio is possible in attempt to tune the protocols performance according to the application criticality level.
\end{abstract}

\section{Introduction}

Vehicular ad hoc network (VANET) is considered as a promising technology for ensuring the safety and the efficiency of intelligent transportation systems (ITS).
A wide variety of safety and comfort applications [7][8] are offered through an extensive exchange of different data amongst connected vehicles. Efficient data dissemination is needed toward the wireless and mobile environment challenges. Therefore, a considerable focus on the data dissemination protocols' design in VANET is required. Tremendous efforts have addressed the data dissemination issues and thereby a large number of protocols have been proposed in the literature.

The performance evaluation of such protocols is essential to enhance their design and confirm their efficiency. Due to the lack of typical methodology used for the performance evaluation of data dissemination schemes in vehicular networks, most of proposed protocols are commonly evaluated and validated through specific simulations' scenarios. This maybe mainly explained by the challenging task of providing sufficient details about the traffic scenarios and the driving behavior to be close as more as possible to the realistic scenario. Indeed, three modeling fields are to be considered in VANET, which are the road and map layout model, the vehicle mobility model and the message dissemination model. The data dissemination modeling is the main purpose of the current work.

Indeed, we present in this paper two variants of protocols, called SEAD1 and SEAD2, that combine a probabilistic and a delay based mechanisms to avoid the famous "Broadcast storm" [27] problem. These protocols are inspired from the SEAD (Simple and Efficient Adaptive data Dissemination) protocol [1]. Their main feature lies in their efficiency and their ability to be adaptively tuned according to the VANET applications' requirements. In fact, 
we strongly believe that an adaptive dissemination protocol is a outstanding design that may ensure the trade-off between the data reachability and the consumption of the limited wireless resources according to the services needs. However, this adaptivity feature needs a deep investigation of the system behavior to subsequently determine the faithful values for the protocol settings, according to the different VANET applications' needs.

Therefore, one of the main contributions in this work is to build an analytic model for the proposed data dissemination protocols. These protocols have been designed to disseminate messages along a road or motorway in one or two directions (upstream/downstream). Messages can be related to safety or less sensitive (advertising, traffic information, etc.) applications. The studied performance metrics are mainly the packet delivery ratio and the redundancy ratio (bandwidth usage). For a complete protocol modeling and evaluation a set of realistic simulations is performed with NS3 simulator.

In short, the aim of the current work through the data dissemination protocols modeling is threefold:

- First, studying in depth the network behavior and thereby having a microscopic vision on the data dissemination process and the protocols design efficiency.

- Second, enabling the network performance management and the protocols parameter tradeoffs. Hence, by knowing the probability and the redundancy ratio behavior we are able to accurately configure our protocol performance according to the application needs. This means that we can predict the accurate amount of redundant packets needed to guarantee a specific ratio of packet reachability.

- Third, formally showing that the proposed protocols converge regardless the network scenarios.

Based on our analysis, we have realized that the local parameter of the SEAD versions should be selected within a certain value range to ensure an efficient setting. Elsewhere, no effect on the protocol performance adaptation could be further noticed.
Hence, by knowing the effective value of the local parameter and then its impact on the system behavior, we become aware of the best metric trade-off which is able to improve the network performance. Thereby, it becomes possible to achieve a defined ratio of data reachability while consuming the minimum network resources. Moreover, in order to confirm the accuracy of the proposed model, we have presented the excellent agreement performed between the numerical and the simulation results.

The remainder of this paper is organized as follows. In Section 2, we present a short overview on data dissemination protocols and we report some previous works that have specifically addressed the performance modeling of their protocols. Section 3 provides a thorough description of the proposed protocols. Section 4 presents in detail the proposed model for the redundancy and the packet delivery ratio. Section 5 discusses the numerical and simulation results under perfect radio environment and shows the protocols performance under realistic radio and traffic pattern. Finally, concluding remarks and future works are presented in Section 6 .

\section{Related works}

\subsection{Data dissemination protocols}

Several researchers have proposed data dissemination protocols dealing with the Broadcast Storm problem. Their common solution is to decrease the number of transmissions by just selecting a set of nodes as forwarders to relay the message further in the network. However, the difference between these solutions relies on the criteria chosen to perform the selection process. Referring to some reviews [4][5][14][19] , we can basically distinguish two categories, on which we are interested, namely, the delay-based and the probability-based techniques.

The main concept of the delay-based strategy is to assign a waiting timer for each receiving vehicle before reforwarding the message. Such timer is cancelled whenever the vehicle receives a redundant message. Hence, only few vehicles will perform their re-transmission process upon the timer expiration. However, the timer calculation and optimization re- 
mains an essential focus for researchers, leading to various number of delay-based data dissemination protocol [3][11][15][16][30][22][23][24] [25]. The same concept was adopted with the probability-based protocols where a defined probability of broadcast is assigned to each receiving node instead of a waiting time. One of the main concern of such data dissemination category is how to define the optimal probability design. This means which network criteria should be taken into account without rising the probability complexity calculation. In this context, we cite the well-known protocol called "weighted p-persistence protocol" in [30] and the "Irresponsible Forwarding" protocol proposed in [18].

Generally, the major parameters taken into account in these strategies are essentially the distance between the source node and the receiver, the message and the transmitter moving direction and the surrounding vehicles' density.

In the following section, we focus on reviewing recent works that have addressed the data dissemination performance especially through analytic models.

\subsection{Performance modeling}

In [20], authors have defined the lower bound of the receiving probability calculated for safety message upon multi-hop communication. This probability was derived from a one hop data reachability presented by a constant probability $p$. The aim of this performance modeling is to discuss the trade-off between $p$, the transmission delay $t$ and inter-vehicle distance $d$. This latter parameter was assumed constant which is not the case in mobile network where the topology changes dynamically.

In [29], two safety message dissemination protocols called "Optimal" and "Global", already proposed in [20], were studied. In order to obtain their timeprobabilistic features, an analytic model in a simple linear network is proposed. In this model, authors have assumed that each broadcast message from a transmitter is received by all the vehicles within its transmission range with a probability $p$. The main performance metric considered is the mean dissemination delay. Based on this model, it seems likely that it is an easy task to compare different protocols.
However, merely few works can be considered under such simple model. Indeed, this model represents exactly the lower bound on the mean dissemination delay for the "Optimal" algorithm and the upper bound on the mean dissemination delay for the "Global" algorithm.

In [12], authors have tackled the performance modeling from another side by assuming that safety messages have higher priority than ones used either for traffic infotainment or other comfort applications. Moreover, authors have defined two types of interferences: external interference is detected whenever the distance between two concurrent transmitters is less than 2 times the transmission range, if this distance is less than the transmission range, the interference is then referred to as an internal interference. Based on this concept, a probability of interference between two nodes is defined. Then, through a birth-death process analysis, the number of concurrent transmissions of lower priority messages, is derived at the steady-state of the system. Also, the percentage of non-successfully received messages due to the interference effect is deduced. Subsequently, the higher priority traffic is determined in the presence of the lower priority traffic. Three performance metrics were considered, namely, the average forwarding distance in one hop, the average number of reached hops and the average number of successfully reached nodes.

The advantage of this work is that the proposed models can be further used for the performance evaluation of data dissemination protocols. Upon this existing work, authors in [13] have built a new analytic model. The main contribution of this model lies in its ability to define the joint probability distribution of the number of messages which are in transmission mode and in backoff process, via 2-D Markov modeling at system steady state.

In [9], authors have discussed safety message dissemination design issues for inter-vehicle communication. To this end, an analytic model was built for an accurate configuration of the transmission probability at each forwarding node. Multi-hop broadcast cycles are considered in order to guarantee that all vehicles within the safety area, where the hazardous event is detected, are informed. Vehicles are supposed to 
move at high speed in one dimensional road. Three performance metrics for the data dissemination service are derived for an accurate setting, which are the probability of successful reception, the average delay and the average number of redundant packet. However, packet collisions are neglected in this work and it's assumed that the safety message is successfully received by all vehicles within the source transmission range.

Besides, authors in [28] have addressed the message generation rate impact on the dissemination performance in terms of end-to-end delay and probability of successful message reception. In this work, one-hop communication scenario is considered since only beacon messages are studied using IEEE 802.11p standard. Unlike other studies, a lossy network is assumed where messages could be lost due to noise and collisions problems. This work could be practical for VANET modeling if it is generalized for other message types while considering a multi-hop communication.

All the aforementioned studies aim to offer an accurate data dissemination modeling as close as possible to the reality. However, some realistic details are still remaining a great challenge in VANET, since they contribute to increase the model complexity level. Therefore, most researchers have addressed the data dissemination modeling by relaxing some network limitations. Their main objective is to build generalized models that can be useful for all dissemination protocols analysis. However, despite the limited impact of some assumptions, such as, the transmission time and the back-off time, other assumptions, like collisions problems, propagation channel model, etc., have most likely a significant effect on the performance modeling. For this reason and in relation with the considered assumptions, most of proposed models remain applicable for a specific category of algorithms.

As a result, even if their accuracies are proven, none of the discussed models could be applied in the current study, since specific assumptions and features should be taken into account in attempt to derive the effective protocol performance. As mentioned above, our focus in this work is to build an accurate analytic model for our data dissemination protocol called "SEAD" which may be adapted to VANET applications' requirements just by tuning a specific local parameter. Thus, faithful values of this parameter should be derived in order to guarantee the best trade-off between the major performance metrics considered in vehicular environment.

\section{Protocols Description}

In this section, we aim to primarily present the key features the two SEAD versions which are distinguished through the adopted reception design. In Fig. 1 and Fig. 2 we present the reception procedure of SEAD1 and SEAD2, respectively.

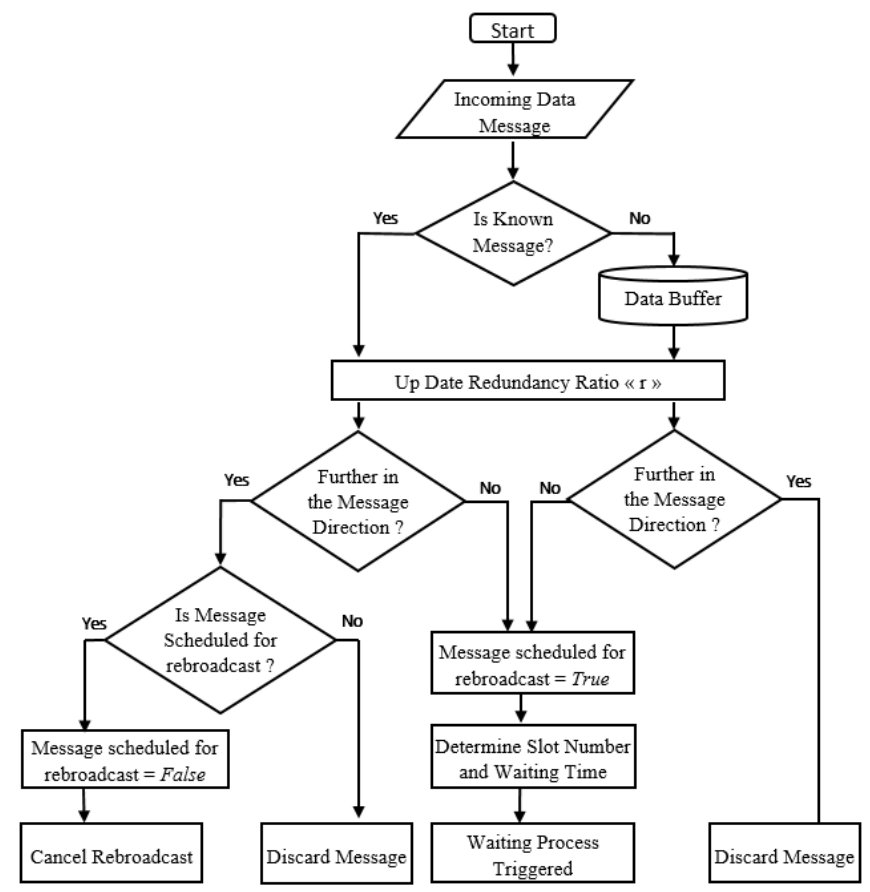

Figure 1: Flow chart of Reception Procedure for SEAD1 Protocol

In both versions of SEAD, two checking phases are adopted. One first check is related to the originality of the message and one second check is about the direction origin of the message with relation to the dissemination direction. 


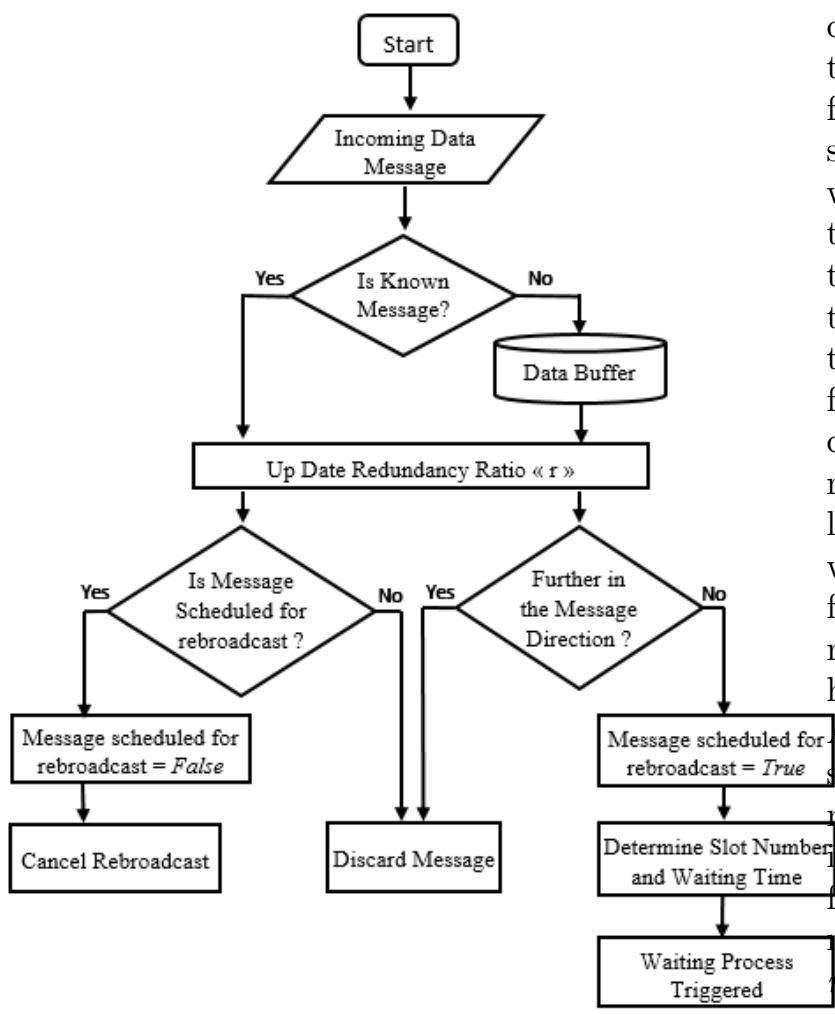

Figure 2: Flow chart of Reception Procedure for SEAD2 Protocol

Therefore, upon receiving a message, the vehicle checks first whether the message's ID is already stored in the data buffer or not. This is performed in order to verify the newness of the message. If the message is new, it is copied in the Data buffer. Otherwise, the received message is considered redundant . At the end of such test, a specific defined parameter called Redundancy Ratio and denoted "r" is updated and the second check procedure is triggered. Noting that the " $\mathrm{r}$ " metric is responsible for measuring the number of received messages (new or duplicated) per new message. It gives the number of times the same message is received by one vehicle which will be later exploited in the rebroadcasting procedure.

For the new received messages, the same procedure is carried out by both SEAD1 and SEAD2 versions. Typically, the new received messages are automati- cally scheduled for rebroadcast. However, SEAD protocol is more selective toward the message suitability for a next retransmission with relation to the dissemination direction. Hence, the message is checked whether it is broadcasted from a node situated further in the message direction or not. If we consider that the message dissemination direction is opposite to the vehicles moving direction, we can say that the receiving node checks whether the message comes from backward or not. The purpose behind this procedure is to determine the relevance of the message regarding the receiver vehicle and hence reducing useless messages. According to SEAD versions design, we assume that a new message may not be useful for data dissemination if it comes from a backward node (downstream) as this means that the message has reached further nodes in the message direction. As a result, no waiting time calculation is needed for uch message that should be discarded. Otherwise, new message received from forward node (upstream), s treated as a relevant information that need to be urther disseminated in the message direction. This nessage must be tagged by the label "scheduled for ebroadcast $=$ True" in order to determine its Slot number and thus assigning its corresponding waiting timer " $W_{t}$ " that is immediately triggered.

Nevertheless, redundant messages are differently trea-ted by SEAD1 and SEAD2. According to SEAD1, a redundant message transmitted from forward (upstream) does not confirm a successful propagation of the message further throughout the network. Thereby, the receiving node schedules the message for an upcoming rebroadcast if it is not already done. Only the reforwarding process of duplicated messages received from downstream should be cancelled. However, in SEAD2, the duplicated message is either discarded or its waiting timer is cancelled regardless from where it comes (from both directions).

For the waiting time calculation, our interest goes to the slotted technique, as the continuous technique has shown its unsuitability for safety messages dissemination, which is highly sensitive to the transmission delay [2]. In particular, we have focused on the "slotted 1-persistence dissemination protocol" (S1PD), since it is considered as the most efficient delay-based strategy upon which further modifica- 
tions and enhancements may be conducted [22] [21]. In short, the main idea of S1PD is to divide the radio range of a transmitter into several segments, denoted "slots". Each segment is then associated to a different waiting time. Vehicles belonging to the farthest segment will be assigned to the shortest time. One of the major limit of S1PD is the local broadcast storm where multiple vehicles belonging to the same segment trigger their transmissions simultaneously, since they are assigned to the same timer. This may result in a high number of collisions and a waste of consumed resources. To cope with this problem, we have added a random extra micro delay, defined in Equation 1, for each transmitter at the application layer. Moreover, this extra delay has shown a great effect to overcome the simulator synchronization effects in low dense network.

$$
\mu_{\text {delay }}=\operatorname{Random}(0,2 \cdot A I F S)
$$

Hence, the waiting time calculated in this work is presented in Equation 2:

$$
W_{t}=\left\lfloor N_{s t} *\left(1-\frac{\min \left(D_{i j}, R\right)}{R}\right)\right\rfloor * \delta+\mu_{\text {delay }}
$$

where $N_{s t}$ is a fixed number of segments, $D_{i j}$ is the relative distance between the transmitter $i$ and the receiver $j, R$ is the average transmission range and $\delta$ is larger than one hop delay including medium access delay and propagation delay. All the numerical values of these parameters are mentioned in Table 1.

For the rebroadcasting procedure is similar for both SEAD1 and SEAD2. Such procedure is triggered when the message's timer expires and no redundant message is received from other forwarders during the waiting process. The message is broadcasted with a new proposed probability of broadcast $p$ in order to efficiently manage the number of transmitters in a segment. The originality of $p$ is that it is function of the defined Redundancy Ratio metric $(r)$, as presented in Equation 3:

$$
p=\frac{2 \alpha}{r_{i}} \cdot p_{\text {prev }}
$$

where " $r_{i}$ " represents the current redundancy ratio value calculated at the sending time "i" and "P $P_{\text {prev }}$ " is the previous calculated probability for the last sent packet. The aim of this correlation, is to adaptively regulate the redundancy ratio in order to maintain a normalized amount of redundancy. Ideally, the expected redundancy is then $2 \alpha$, where $\alpha$ is a configurable parameter of the protocol.

It is merely obvious that $r$ increases when the vehicles' density increases, since more vehicles are involved in the dissemination process. That is why the redundancy is inversely proportional to the forwarding probability. Based on the forwarding probability design we can deduce that, in addition to the message dissemination direction considered to determine the re-broadcast possibility and the distance parameter used for computing the waiting time, both SEAD versions are able to implicitly take into account the vehicles' density through the redundancy parameter without need for beacons exchange containing neighbors' information [3]. This is considered as one of their main key features.

From another side, the proposed protocols seem capable to fit all types of applications. Indeed, through the parameter $\alpha$, we are able to adjust the rate of data reachability according to the application's requirements. This adaptivity feature leads to improve data dissemination efficiency by reducing the amount of non-useful redundant messages. In fact, in a lossy network, redundant packets are essential to ensure the network reliability. Yet, whenever this amount exceeds a particular threshold, it becomes the major cause of collisions and resources consumption waste.

Through this solution, we try to define the optimal (minimum) amount of redundancy able to guarantee the required packet delivery ratio. Since they meant to address passenger safety and to decrease the loss of life, safety messages have more priority and require high reachability throughout the network. Whereas, message addressing the passenger comfort are more tolerant in terms of data reachability and even in transmission delay. Thus, thanks to the proposed SEAD versions, the network resources consumption is more balanced according to the application needs. An accurate tradeoff between the redundancy ratio and the packet delivery ratio using the SEAD versions is essential to determine the exact values of $\alpha$ parameter and hence a proper setting of the adap- 
tive protocol. For this reason, a thorough study of the considered metrics is presented in the next section, via an analytic model.

\section{4 analytic Model}

In this section, we propose to evaluate the performance of one of the the proposed protocols, since they are too close in their design, through an analytic model. In particular we choose SEAD1 protocol as it is more general and we simply refer to it as SEAD throughout this section. This model aims to study two key metrics: the redundancy ratio and the packet delivery ratio (PDR). As previously defined, the redundancy ratio represents the mean number of receptions of the same packet. Whereas, the packet delivery ratio is the average proportion of nodes/vehicles that received a disseminated packet. This ratio is equivalent to the mean proportion of new packets received by one vehicle. Before explaining the proposed model, we primarily focus, in the following paragraph, on describing the considered scenario.

a) Scenario: As depicted in Fig. 3, we assume the following scenario. The transmitter, represented with a black disk, re-forward a received message. All nodes situated behind the transmitter (downstream nodes) and within its transmission range, represented with grey disks, successfully receive the message. Indeed, the radio range, denoted $R$, is assumed to be perfect in which only collisions are considered as the cause of transmission failure. Thus in the absence of collisions, all nodes situated at a distance less than $R$ from the transmitter successfully receive the packet.

We divide the radio range into $N_{s t}$ equal segments. In each segment, we assume the presence of $N$ vehicles ( $N=3$ in the figure). It should be noted that $N$ may be a deterministic or a random variable. In this case, all different metrics derived throughout our analysis have to be conditioned with the distribution of $N$. There is no assumption about the location of the vehicles within their segments.

The obtained segments are numbered in a decreas- ing order, from $N_{s t}$ to 1 . The farthest segment matches to the lowest number, which is 1 . According to both SEAD versions design, nodes belonging to segment 1 have the highest priority and try to re-broadcast the packet first as they have the shortest waiting time. Each node in this segment re-broadcasts the packet with a probability $p$ independently from each other. If the transmissions from the nodes in segment 1 fail, then nodes belonging to segment 2 try to transmit, and so on.

b) Redundancy Ratio: The mean redundancy ratio, denoted Redundancy, is defined as:

$$
\begin{aligned}
& \operatorname{Redundancy}(N, p) \\
& =\beta(N, p) \cdot \mathbb{E}_{(N, p)}\left[r_{\text {seg }} \mid r_{\text {seg }}>0\right]
\end{aligned}
$$

The random variable $r_{\text {seg }}$ is the amount of redundancy generated by vehicles belonging to a transmitting segment. Its distribution depends on the parameters $N$ and $p$. These parameters appear as indexes of the expectation. Note that, the transmitting segment does not necessarily correspond to the farthest segment, as all vehicles belonging to the last segment could cancel their re-transmissions with a probability $1-p$. The parameter $\beta(N, p)$ takes into account the fact that re-transmissions are not necessarily generated by the farthest segment. In this case, the distance between two successive transmitting segments (1 hop size) is smaller than the radio range. Thereby, the average number of segments from which a typical vehicle receives the transmission is greater than 2. Exactly, it is given by $\beta(N, p)$. In practice, the SEAD protocol versions are designed to reach a certain redundancy, equal to $2 \alpha$, by adapting the probability of broadcast $p$. Notice that the factor 2 designates the fact that a node receives packets from transmitters belonging to different segments. As in most of the cases, a simple road is considered and a vehicle may receive the packet from two segments (upstream and downstream).

When the targeted redundancy is reached, the quantity Redundancy $(N, p)$ should correspond to the configured $2 \alpha$ value. Notice also that the protocol modifies the rebroadcasting probability 
$p$ only when at least one packet is received (i.e. $\left.r_{\text {seg }}>0\right)$. As a result, the conditional expectation $\mathbb{E}_{(N, p)}\left[r_{\text {seg }} \mid r_{\text {seg }}>0\right]$ is then more accurate to model the protocol behavior.

In the following paragraphs, we primarily focus on deriving the average number of receptions per one packet generated by one segment $\left(\mathbb{E}_{(N, p)}\left[r_{\text {seg }} \mid r_{\text {seg }}>\right.\right.$ $0])$ and the number of segments from which a vehicle receives a packet $(\beta(N, p))$. Second, we highlight the limited values of redundancy metric that shows a lower and an upper bound. A generalization of redundancy model is then deduced. A particular study of the asymptotic behavior of the redundancy is presented when $N \rightarrow+\infty$. Thereafter, we move to propose an estimation of the PDR metric. Finally, two propositions that summarize the analytic results are given at the end of this section.

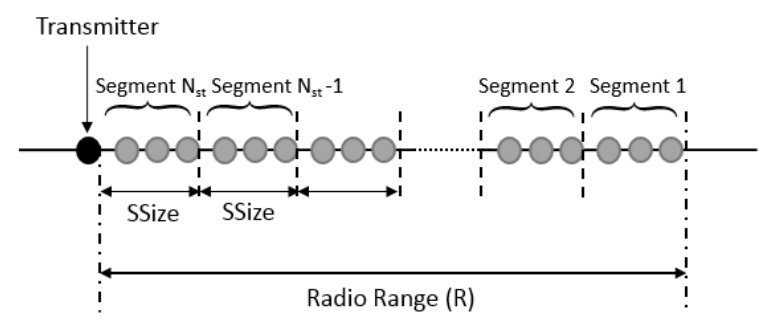

Figure 3: analytic model scenario

\subsection{Redundancy generated by a seg- ment}

In this paragraph, we propose to model and evaluate the number of re-transmissions generated by one segment. In particular, we consider the segment that is in charge of re-transmitting the packet. According to the protocol design, this segment should be the last one. However, when vehicles belonging to the last segment fail their transmissions, then next segment is involved and so on.

The number of re-forwarders in the segment from where the packet is transmitted, denoted $N_{T}$, follows a binomial distribution with parameters $(N, p)$.

Thus, $N_{T}$ transmissions are exactly performed from this segment, however, not all of them will be successfully received by neighboring vehicles. As $N_{T}$ nodes compete for the medium at the same time and apply the CSMA/CA mechanism, their transmissions may suffer from collisions. In fact, a collision occurs as soon as two re-transmitters have chosen the same back-off. In this case, we assume that the frames are lost, and the corresponding packets are not counted as redundant.

The probability of non-collision of a particular transmission, generated by a vehicle within the segment, is given by $\left(1-\frac{1}{C W}\right)^{N_{T}-1}$ where $C W$ is the size of the contention window (equals to 15 in $802.11 \mathrm{p})^{3}$. In other words, it is the probability that the $N_{T}-1$ other transmitters within the same segment have chosen back-off values different from that selected by this vehicle.

In the current model, we did not take into account the other phenomena that may have an impact on packet reception success ( the radio environment for instance). But we shall see later, through realistic simulations, that collisions are the major phenomena that influence the number of receptions and therefore the protocol performance.

On the basis of the different assumptions, the redundancy ratio is equal to the number of transmissions (from $N_{T}$ vehicles) that do not collide in a given segment. It is defined as follows:

$$
\begin{aligned}
\mathbb{E}_{(N, p)}\left[r_{\text {seg }}\right]= & \sum_{k=1}^{N} \mathbb{E}\left[r_{\text {seg }} \mid N_{T}=k\right] \mathbb{P}_{(N, p)}\left(N_{T}=k\right) \\
= & \sum_{k=1}^{N} \mathbb{E}\left[\sum_{i=1}^{k} \mathbb{1}_{\text {tx from veh } i \text { does not collide }} \mid N_{T}=k\right] \\
& \times\left(\begin{array}{l}
N \\
k
\end{array}\right) p^{k}(1-p)^{N-k} \\
= & \sum_{k=1}^{N} k\left(1-\frac{1}{C W}\right)^{k-1}\left(\begin{array}{l}
N \\
k
\end{array}\right) p^{k}(1-p)^{N-k} \\
= & N p\left(1-\frac{p}{C W}\right)^{N-1}
\end{aligned}
$$

$\mathbb{1}$. is the indicator function equals to 1 if the indexed condition is true and 0 otherwise. As previously explained, the conditional expectation fits more

\footnotetext{
${ }^{3}$ In our case (broadcasting), $C W$ remains constant as no acknowledgment nor re-transmissions mechanisms are deployed.
} 
our protocol design, thus we obtain the following:

$$
\begin{aligned}
\mathbb{E}_{(N, p)}\left[r_{\text {seg }} \mid r_{\text {seg }}>0\right] & =\frac{\mathbb{E}_{(N, p)}\left[r_{\text {seg }} \mathbb{1}_{\text {seg }}>0\right]}{\mathbb{P}_{(N, p)}\left(r_{\text {seg }>0}\right)} \\
& =\frac{\mathbb{E}_{(N, p)}\left[r_{\text {seg }}\right]}{\mathbb{P}_{(N, p)}\left(r_{\text {seg }}>0\right)}
\end{aligned}
$$

The computation of such denominator, $\mathbb{P}_{(N, p)}\left(r_{\text {seg }}>0\right)$, is not trivial. The condition $r_{\text {seg }}>0$ holds when at least one vehicle, among the $N_{T}$, picks up a backoff value which is different from those picked by other vehicles. This boils down to an enumerative combinatorial problem. To make it simple let's consider the following problem: we put $N_{T}$ balls into $C W$ urns, what is the probability that at least one urn contains exactly one ball? If $N_{T}=k$, the solution of such problem relies on the following probability, denoted $e c(.,$.$) (ec stands for$ enumerative combinatorics):

$$
\begin{aligned}
e c(C W, k)= & \frac{1}{C W^{k}} \sum_{j=1}^{\min (k, C W-1)}\left(\begin{array}{l}
k \\
j
\end{array}\right) \frac{(-1)^{j+1} C W !}{(C W-j) !}(1) \\
& \times(C W-j)^{k-j}
\end{aligned}
$$

when $k>0$ and $e c(C W, 0)=0$.

By taking into account the $N_{T}$ distribution, we get:

$$
\mathbb{P}_{(N, p)}\left(r_{\text {seg }}>0\right)=\sum_{k=1}^{N} e c(C W, k)\left(\begin{array}{l}
N \\
k
\end{array}\right) p^{k}(1-p)^{N}(\mathrm{~T} 2)
$$

\subsection{Computation of $\beta(N, p)$}

In this work, we propose an approximation of $\beta(.,$. as follows:

$$
\beta(N, p)=\frac{R}{\mathbb{E}_{(N, p)}[H L \mid H L>0]}
$$

where $R$ is the radio range, and $H L$ the random variable representing the hop length, i.e. the distance between two successive re-transmitting segments. $H L$ is formally defined as the mean distance between two successive segments that successfully transmit the packet. A simple estimation of its average is given by:

$$
\begin{gathered}
\mathbb{E}_{(N, p)}[H L]=\sum_{k=1}^{N_{s t}}\left(\left(N_{s t}-k\right) * \text { SSize }+\frac{\text { SSize }}{2}\right) \\
\times \mathbb{P}_{(N, p)}\left(r_{\text {seg }}>0\right) \mathbb{P}_{(N, p)}\left(r_{\text {seg }}=0\right)^{k-1} \\
=\text { SSize } \\
{\left[N_{s t}+\left(\frac{1}{2}-\frac{1}{\mathbb{P}_{(N, p)}\left(r_{\text {seg }}>0\right)}\right)\right.} \\
\left.\times\left(1-\mathbb{P}_{(N, p)}\left(r_{\text {seg }}=0\right)^{N_{s t}}\right)\right]
\end{gathered}
$$

In this equation, the term $\mathbb{P}_{(N, p)}\left(r_{\text {seg }}>\right.$ $0) \mathbb{P}_{(N, p)}\left(r_{\text {seg }}=0\right)^{k-1}$ represents the probability of successful packet rebroadcast from the $k^{\text {th }}$ segment. SSize is the size of a segment. The hop length is then estimated by $\left(N_{s t}-k\right) * S$ Size $+\frac{\text { SSize }}{2}$. We implicitly consider that $H L=0$ if all segments fail in re-transmitting the packet. Similarly to the previous calculation, this expectation makes sense when, at least, there is one segment that re-transmits properly the packet. Consequently, a conditional expectation of $H L$ is considered, given that $H L>0$ :

$$
\mathbb{E}_{(N, p)}[H L \mid H L>0]=\frac{\mathbb{E}_{(N, p)}[H L]}{\mathbb{P}_{(N, p)}(H L>0)}
$$

It is obvious to say that a non-successful transmission for a given hop amounts to a non-successful transmission for all its constituting segments. The probability that the transmission fails for a given segment is $\mathbb{P}_{(N, p)}\left(r_{\text {seg }}=0\right)$. Thereafter, as the probability of collisions is independently computed between the different segments, we obtain the following expression:

$$
\mathbb{P}_{(N, p)}(H L>0)=1-\mathbb{P}_{(N, p)}\left(r_{\text {seg }}=0\right)^{N_{s t}}(17)
$$

\subsection{Limit values of the redundancy and asymptotic behavior}

The redundancy, as given by Equation (4), cannot be arbitrarily high or low. For instance, if the number of vehicles (denoted $N$ ) within a segment is high and the probability $p$ is close to 1 , the number of transmitters will be high and many transmissions will collide, leading to an upper limit of redundancy that shoud not be exceeded. On the other hand, it admits also a lower limit that will be discussed below. 
a) Limit Values: From a mathematical point of view, the quantity Redundancy $(N, p)$ is a function of $p \in[0,1]$ that takes its values in a sub-interval of $[1, N]$. This interval, that depends on $N$, is denoted $\left[\operatorname{red}_{\min }(\mathrm{N}), \operatorname{red}_{\max }(\mathrm{N})\right]$.

With regard to the minimum and maximum, they are formally computed as follows:

$$
\begin{aligned}
& \operatorname{red}_{\text {max }}(N)=\max _{p \in[0,1]} \operatorname{Redundancy}(N, p)(18) \\
& \operatorname{red}_{\text {min }}(N)=\min _{p \in[0,1]} \operatorname{Redundancy}(N, p)(19)
\end{aligned}
$$

The two noticed bounds on the redundancy are plotted in Fig. 4. We observe that for small values of $N$, the maximum and minimum redundancy increase as long as more transmitters can be involved to broadcast the packet. When $N$ reaches approximately $15-20$, the maximum and minimum reach a plateau and remain almost constant. In other words, the minimum/maximum redundancies seem to tend to a limit level when $N \rightarrow+\infty$.

We have to note here that the number of vehicles within 100 meters could be considered as infinity for 20 vehicles. In fact, all vehicles have to respect "The two-second rule" [6] which is the minimum timing by which a driver may preserve a safe following distance with the car in front. Thus, a driver may have enough time to react. As we are considering a Highway scenario, generally the average speed is $20 \mathrm{~m} / \mathrm{s}$ and then the safety distance is $40 \mathrm{~m}$, which means we can have at most 3 vehicles per segment for each lane.

Impact on $\alpha$ The parameter $\alpha$ is the desired redundancy ensured by the protocol. The probability to retransmit a packet $p$ is a function of $\alpha$ and the measure of the redundancy in the previous transmission as given by Equation (3). On the bases of these curves, we are aware about the $\alpha$ value range (minimum and maximum value) for which a proper setting of the protocol is possible. For an $\alpha$ over the maximum value, the protocol achieves a steady behavior and no more re- dundancy can be reached. This is explained by the fact that the possibilities of broadcast are increased and thereby more collisions are experienced. For example, in our simulation we can observe that for $65 \mathrm{veh} / \mathrm{km}$ (which means roughly 6 vehicles per segment) we cannot have more than 4 redundant packets, as depicted in Fig. 7(a).

b) Asymptotic Behavior: In order to deeply study the redundancy limit, we focus on investigating the asymptotic behavior of the conditional redundancy (when $N \rightarrow+\infty$ ). As we can observe from Fig. 4, the limit is reached approximately when $N>12$ for $\operatorname{red}_{\max }(N)$, and $N>30$ for $\operatorname{red}_{\min }(N)$. Moreover, we assume that $p$ can be simply approximated by $\frac{c}{N}$ when $N$ increases, where $c$ is a positive constant. This assumption is legitimate. According to SEAD design, $p$ values are continuously varied in such a way to reach the desired amount of redundancy $2 \alpha$. In other words, based on the probability of broadcast, SEAD protocol tries to adapt the number of re-transmitters which are able to guarantee the required redundancy. Thus, SEAD tends to choose a particular value of $p$ that depends on the number of transmitters $\mathbb{E}\left[N_{T}\right]=p N=c$ which leads to $\mathbb{E}_{(N, p)}\left[r_{\text {seg }} \mid r_{\text {seg }}>0\right]$.

On the basis of this assumption, $N_{T}$ follows a discrete Poisson distribution with parameter $c$, and $\mathbb{E}_{(N, p)}\left[r_{\text {seg }}\right] \rightarrow c e^{-\frac{c}{C W}}$ when $N$ tends to infinity. To compute the conditional expectation we set:

$$
\mathbb{P}_{\text {lim }}\left(r_{\text {seg }}>0\right)=\lim _{N \rightarrow+\infty, p \approx \frac{c}{N}} \mathbb{P}\left(r_{\text {seg }}>0\right)
$$

As ec $(C W, k)=e c(C W, C W-1)$ when $k \geq C W-$ 1, we get:

$$
\begin{aligned}
& \mathbb{P}_{\text {lim }}\left(r_{\text {seg }}>0\right) \\
= & \sum_{k=1}^{C W-1} e c(C W, k) \frac{c^{k}}{k !} e^{-c} \\
& +\sum_{k=C W}^{+\infty} e c(C W, C W-1) \frac{c^{k}}{k !} e^{-\alpha} \\
= & 1-\sum_{k=0}^{C W-1}(e c(C W, C W-1)-e c(C W, k)) \frac{c^{k}}{k !}(2 \mathrm{I})
\end{aligned}
$$




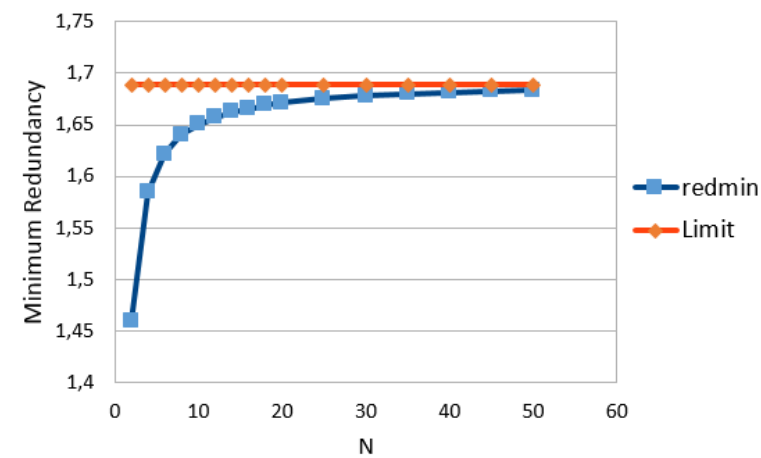

(a) $\operatorname{red}_{\min }(N)$

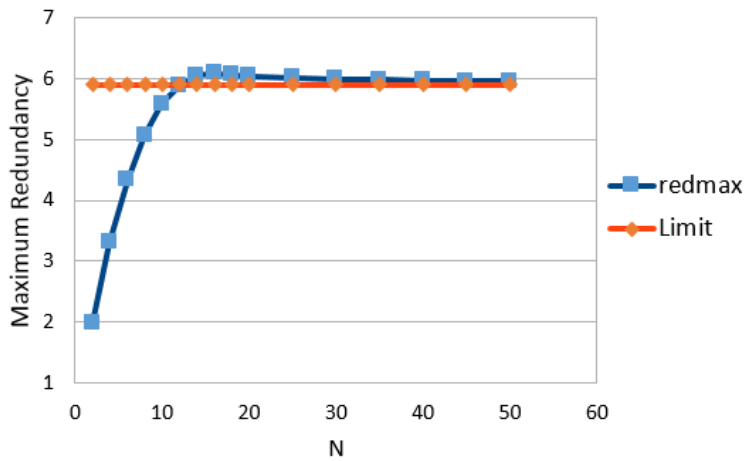

(b) $\operatorname{red}_{\max }(N)$

Figure 4: Maximum and minimum redundancy values and asymptotic behavior

Finally, we obtain when $p \approx \frac{c}{N}$ and $N \rightarrow+\infty$ :

$$
=\frac{\lim _{N \rightarrow+\infty, p \approx \frac{c}{N}} \mathbb{E}_{(N, p)}\left[r_{\text {seg }} \mid r_{\text {seg }}>0\right]}{1-\sum_{k=1}^{C W-1}(e c(C W, C W-1)-e c(C W, k)) \frac{c^{k}}{k !} e^{-c}}
$$

The computation of $\lim _{N \rightarrow+\infty, p \approx \frac{c}{N}} \beta(N, p)$ is straightforward. It is simply a matter of replacing $\mathbb{P}_{(N, p)}\left(r_{\text {seg }}>0\right)$ by $\mathbb{P}_{\text {lim }}\left(r_{\text {seg }}>0\right)$ in Equations (15) and (17).

In Fig. 4, we added two horizontal lines corresponding to the minimum and maximum limit redundancy (minimum/maximum for all possible values of $c$ ). The minimum and maximum redundancy are 1.68 and 5.88, respectively. It clearly appears that these two values perfectly fit with the limit of the two redundancy curves.

\subsection{Packet Delivery Ratio}

In this Section, we propose to evaluate the packet delivery ratio (PDR) computed as the mean proportion of nodes that properly receive a given packet. Under a perfect radio range, we assume that if the dissemination of a packet fails at a certain hop, all nodes situated beyond the reached hop will never receive this packet. This means that the packet dissemination stops once it fails at certain dissemination distance. The reasons for which the dissemination may stop are mainly: i) severe collisions experienced by (22) all transmissions generated from the next forwarders ii) absence of re-transmitters due to the decision of transmission cancellation by all potential forwarders

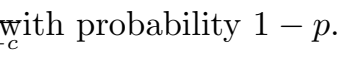

Let's consider $\mathrm{NH}$ the random variable that designates the number of hops really travelled by the packet (more precisely it corresponds to the number of segments for which at least one transmission succeeds). $H$ is the expected number of hops. It is assumed to be known in relation with the application requirements (i.e. until which distance the information carried out by the packet is valuable for a given application). The PDR estimation is given by:

$$
P D R=\frac{\mathbb{E}_{(N, p)}[N H]}{H}
$$

Whereas, the computation of $\mathbb{E}_{(N, p)}[N H]$ is performed as follows. First, we need to determine the dissemination failure probability from a given hop $\left(\mathbb{P}_{(N, p)}(H L=0)\right)$. This probability is already computed and it is shown that it is independent and equally distributed at each hop. Indeed, collisions are independent from one hop to another and packet forwarding decision is independent from one vehicle to another. Assuming that the first transmission always succeeds, as it is transmitted by the source $(N H \geq 1)$, we get for $k<H$ the expression below: 


$$
\begin{array}{cl}
\mathbb{P}_{(N, p)} & (N H=k) \\
= & \mathbb{P}_{(N, p)}(H L=0)\left(1-\mathbb{P}_{(N, p)}(H L=0)\right)^{\left.k-\lambda^{-} 24\right)}
\end{array}
$$

As $N H \leq H$, thus

$$
\begin{array}{ll}
\mathbb{P}_{(N, p)} & (N H=H) \\
= & 1-\sum_{k=1}^{H-1} \mathbb{P}_{(N, p)}(H L=0)\left(1-\mathbb{P}_{(N, p)}(H L=0)\right) \\
= & \left(1-\mathbb{P}_{(N, p)}(H L=0)\right)^{H-1}
\end{array}
$$

the main factors that influence the network behavior and the dissemination efficiency.

The considered simulation platform is build upon NS3 [17] simulator. It is a network simulator in which a full implementation of VANET protocol stack is given. In particular, the IEEE 802.11p standard [10] is integrated for the physical layer. In this context, we have implemented SEAD1 and SEAD2 protocols and ${ }^{-1}$ performed two basic simulation sets under different parameters configuration, summarized in Table 1.

Therefore, $\mathbb{E}[N H]$ is the mean of this truncated geometric distribution (until $H$ ):

$$
\begin{aligned}
\mathbb{E}_{(N, p)} & {[N H] } \\
= & \sum_{k=1}^{H-1} k \cdot \mathbb{P}_{(N, p)}(H L=0)\left(1-\mathbb{P}_{(N, p)}(H L=0)\right)^{k-1} \\
& +H \cdot\left(1-\mathbb{P}_{(N, p)}(H L=0)\right)^{H-1} \\
= & \frac{1-\mathbb{P}_{(N, p)}(H L>0)^{H}}{1-\mathbb{P}_{(N, p)}(H L>0)}
\end{aligned}
$$

(25) The first set of simulations attempts to confirm the aforementioned analysis. Hence a comparative study between the numerical results and the simulation results is fulfilled while considering identical environmental settings, such as the vehicles position and the radio environment, as those adopted in the model.

Whereas, the other set of simulations is performed under more realistic environment for a complete study of the proposed protocols. Via a micro-traffic simulator, called "SUMO - Simulation of Urban Mo(26) bility" [26], we have generated a realistic mobility trace in a highway length of $6 \mathrm{~km}$. An overtaking behavior and lane change possibility are allowed in a three-lanes road at a maximum driving speed of $20 \mathrm{~m} / \mathrm{s}$. The transmission power is adjusted to approximately achieve 700 meters of communication range, since Nakagami-m propagation loss model is assumed. Adding to that, we have evaluated the protocol scalability while varying the vehicles' density from 8 vehicles $/ \mathrm{km}$ to 99 vehicles $/ \mathrm{km}$. Note that each illustrated result is an average of 20 runs (performed under the same scenario but using different random seeds). The Confidence intervals are not shown in the Figures as they are negligible. For both simulation scenarios we set the different MAC layer parameters as follows: the Contention Window (CW) value is constant, the slot time, the SIFS (Short Interframe Space) and the DIFS (DCF Interframe Space) are equal to $13 \mu s, 32 \mu s$ and $58 \mu s$, respectively. Regarding the delay based mechanism, we fix the total number of segments $N_{\text {st }}$ to 7 (one segment per 100 $\mathrm{m}$ ) and we set a $\delta$ shift of $4 \mathrm{~ms}$ for each segment (so the timer is $0 \mathrm{~ms}$ for the farthest segment and $6 * 4 \mathrm{~ms}$ for the closest one). For the application scenario, we configure each source node to periodically generate a new message every second. The message size is 
settled to 500 bytes.

The main performance metrics considered for the protocol evaluation and validation are as follows:

- Redundancy Ratio (R): represents the average amount of received messages per one original message for one vehicle.

Table 1: Simulation parameters

\begin{tabular}{|c|c|c|}
\hline & Parameters & Specificatic \\
\hline Perfect & Radio model & Perfect \\
\hline \multirow{4}{*}{ Scenario } & Mobility trace & Static \\
\hline & Simulation duration & 500 second \\
\hline & Nbr of source nodes & 1 source \\
\hline & Vehicles' density & $65 \mathrm{veh} / \mathrm{km}$ \\
\hline Realistic & Radio model & Nakagami \\
\hline \multirow[t]{5}{*}{ Scenario } & Mobility trace & Dynamic \\
\hline & Mobility generator & SUMO \\
\hline & Simulation duration & $100 \mathrm{~s}$ \\
\hline & Nbr of source nodes & 5 \\
\hline & Vehicles' density & 8 to $99 \mathrm{Ve}$ \\
\hline \multirow{3}{*}{$\begin{array}{l}\text { Phy layer } \\
\text { IEEE } 802.11 p\end{array}$} & Frequency band & $5.9 \mathrm{GHz}$ \\
\hline & Bandwidth & $10 \mathrm{MHz}$ \\
\hline & Transmission range & $\sim 700 \mathrm{~m}$ \\
\hline \multirow[t]{5}{*}{ Mac layer } & Bit rate & $6 \mathrm{Mbit} / \mathrm{s}$ \\
\hline & CW & \\
\hline & Slot time & $13 \mu \mathrm{s}$ \\
\hline & SIFS & $32 \mu \mathrm{s}$ \\
\hline & DIFS & $58 \mu \mathrm{s}$ \\
\hline \multirow{2}{*}{$\begin{array}{l}\text { Delay based } \\
\text { technique }\end{array}$} & $\delta$ & $4 \mathrm{~ms}$ \\
\hline & $N_{s t}$ & 7 \\
\hline \multirow[t]{5}{*}{ Application } & Highway length & $6000 \mathrm{~m}$ \\
\hline & Max speed & $20 \mathrm{~m} / \mathrm{s}$ \\
\hline & Data packet frequency & $1 \mathrm{~Hz}$ \\
\hline & Data packet size & 500 bytes \\
\hline & Number of run & 20 \\
\hline
\end{tabular}

- Packet Delivery Ratio (PDR): measures the data reachability rate by comparing the average number of original packets successfully received by a vehicle to the total number of generated messages.

- Forwarding Ratio (FR): represents the proportion of vehicles involved in the dissemination process of a particular packet, compared to the total vehicles present in the network.

- End-to-End Delay (E2EDelay): calculates the average difference between the generation time of a data packet by the source vehicle and the $\mathrm{m}$ reception time of this packet by the last reached vehicle in the network.

- Link Load (bit/s): measures the average of broadcast traffic (in terms of bits) received by each vehicle over a unit of time.

- Packet Drop Ratio : the average amount of erroneous received packets by a vehicle compared to the total received packets.

\subsection{Performance Evaluation with a Perfect Radio Range}

As mentioned before, the goal behind this section is to confirm that analytic results are in agreement with the simulation results given the same environmental details presented in Table 1 . Thus we consider SEAD1 protocol and we refer to it by SEAD protocol for simplicity. Only, the Redundancy and PDR are studied, since they are supposed to be the key performance metrics, through which we can manage the protocol performance to fit VANET applications' requirements.

A constant number $N$ of vehicles is considered within each segment. The radio range is supposed to 


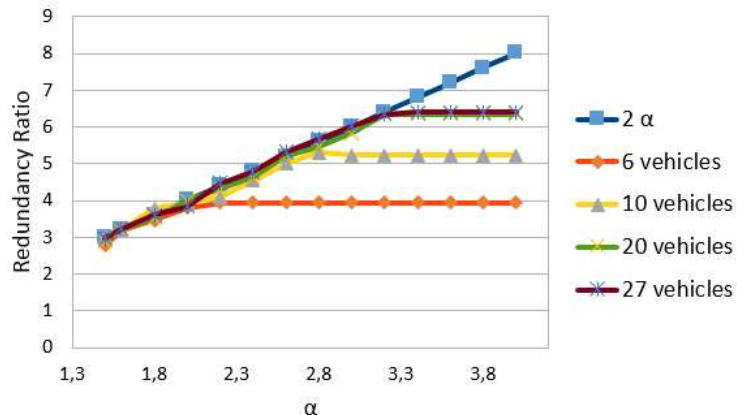

(a) Redundancy

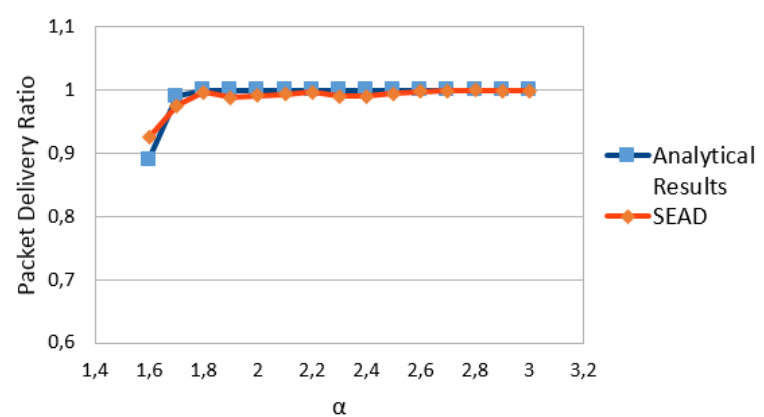

(b) PDR

Figure 5: Comparison between simulation and analytic results for SEAD protocol: Redundancy and PDR

be perfect and is splitted into $N_{s t}$ segments. Therefore, the main reasons for packet loss are either collisions (due to simultaneous transmissions) or no performed transmission (due to the fact that all potential retransmitters have decided to not forward the packet with probability $1-p$ ).

a) Redundancy: Regarding the redundancy metric, we carried out simulations over one hop. We count the number of receptions for each vehicle in the radio range and we compute the average. In Fig. 5(a), we compare the redundancy obtained through NS3 simulations and the exactly desired redundancy $(2 \alpha)$ for $N=6,10,20$ and 27 (randomly chosen values), and for $\alpha$ varying from 1.5 to 4 . We observe that the mean redundancy is exactly equal to $2 \alpha$ in a particular interval. The lower bound of this interval is approximately 1.5 whatever the value of $N$. However, the upper bound level depends on the $N$ value. For instance we get 4 for $N=6,5.2$ for $N=10$ and 6.3 for both $N=20$ and $N=27$. The corresponding theoretical values may be observed in Fig. 4(b) which is generated for one hop. We can observe that the analytic values are 4.33 for $N=6,5.57$ for $N=10$ and 6 for both $N=20$ and $N=27$. It appears that simulation and theoretical are in a good agreement. Nevertheless, a small difference is observed. This may be explained by the transmission delay factor which is neglected in our model: due to the distance separating vehicles, two nodes may detect the transmission from each other even if they have chosen the same backoff value.

As we notice from Fig. 5(a), SEAD protocol does not exceed the upper bound of redundancy even for high $N$ value. This phenomena amounts to the fact that the protocol attempts to increase the broadcast probability $p$ in order to reach higher redundancy which leads to a high number of collisions.

On the other hand, SEAD protocol should not be configured to exhibit redundancy less than the lower bound. The explanation of this behavior is less intuitive and have been investigated through a deep analyze of NS3 traces. When the desired redundancy is less than the lower bound $\operatorname{red}_{\min }(N)$, the protocol becomes in transitional regime and does not converge to a steady state. In fact, whenever $2 \alpha<\operatorname{red}_{\min }(N)$, receiving nodes significantly decrease $p$ in order to reach the desired redundancy. This may induce to decrease the hop length $(H L)$, as long as, the probability of broadcast within the last segment becomes very low. As a result, the number of hops increases and hence the redundancy increases. Therefore, the protocol is still not able to reach the redundancy $2 \alpha$ and needs to decrease even more $p$. At some point, $p$ will become too small to guarantee packets transmission (i.e. $\mathbb{P}_{(N, p)}\left(r_{\text {seg }}=0\right)$ increases) which re- 
sults in low level of PDR for small value of $\alpha$. After a while, packets transmission is stopped and no data is disseminated at all in the network.

Therefore, NS3 simulation results confirm the existing of a particular range of $\alpha$ parameter for which the protocol SEAD is stable and capable to be adapted in order to exactly achieve a redundancy of $2 \alpha$. This range perfectly fits to the ones given by the analytic study.

b) PDR: In Fig. 5(b), we plot the PDR obtained by simulations and that given by Equation (23). In this case, roughly 10 hops have been simulated with $N=6$. The $\alpha$ value is only considered in the stable interval $\left(\alpha \in\left[\operatorname{red}_{\min }(N), \operatorname{red}_{\max }(N)\right]\right)$. We notice that the two curves fit with each other, starting from 0.88 to quickly reach 1.0 . This result shows a good agreement between the numerical and simulation results. Thus, it can be concluded that, the proposed model and its calculations are effective ways to accurately evaluate the PDR of the studied protocol.

The theoretical values can be then used for the protocol configuration, by choosing the accurate value of $\alpha$ that is able to fulfill the application requirements in terms of data reachability (PDR). In order to deploy comfort application, a certain amount of data loss can be tolerated against an important gain in terms of bandwidth saving. Thus an efficient data dissemination for more critical packet is then ensured. For instance, it is worth to choose $\alpha=1.65$ to ensure $90 \%$ of data reachability for advertisement dissemination while saving $25 \%$ of bandwidth consumption. In fact, $\alpha$ remains at a low level which leads to a low amount of packet redundancy. On the other hand, we need choose $\alpha>1.8$ to ensure a total PDR equal to $100 \%$ for safety applications.

\subsection{Performance Evaluation under Realistic Radio Models and Traf- fic Pattern}

The aim through this section is twofold. First, showing the robustness of SEAD protocol adaptiv- ity, while considering realistic radio channel and mobility pattern. Second, highlighting the protocol efficiency for safety message dissemination under different network density. Notice that, throughout this part of work, we distinguish between the two versions inspired from the original protocol, denoted SEAD1 and SEAD2 with relation to the message originator position. In brief, if only redundant message coming from upstream (one side) are discarded, we are talking about SEAD1. Elsewhere, we call SEAD2, the SEAD protocol version in which redundant messages are systematically discarded, either they are coming from upstream or downstream (two sides). The disseminating protocol picked for the performance comparison, is the delay based technique S1PD, based on which we have built our protocols. Comparisons are conducted through the relative scenario described in Table 1.

a) The Protocols Adaptivity Feature: The adaptive feature of SEAD protocol versions presents twofold meaning: local adaptivity and global adaptivity. From one side, thanks to the design of the probability of broadcast formula, each vehicle is able to locally adapt its rebroadcasting ability according to the current network state. From the other side, the protocols performance within the entire network, in terms of data reachability, may be adapted in accordance with the application's requirements (safety or comfort) by tuning the $\alpha$ parameter.

For the sake of confirming the SEAD versions local adaptivity, we show in Fig.7(a) the evolution of the redundancy ratio with regard to the vehicles' density. As we can see, when using S1PD protocol for message dissemination, the redundancy ratio continuously increases with the vehicles' density. Whereas, the redundancy ratio reaches a steady state in both versions SEAD1 and SEAD2 from a density of $50 \mathrm{veh} / \mathrm{km}$ and $70 \mathrm{veh} / \mathrm{km}$, respectively. Although the increasing number of vehicles, the redundancy ratio remains constant. This reveals the ability of transmitters to readjust their transmission suitability according to the network density. Besides, the originality of these protocols lies in the redundancy parameter which is inte- 
grated in the probability of broadcast. Thus vehicles are aware of their surrounding density without need for extra message exchanging (beacons messages management). This awareness may be also noticed in low dense network, in particular for SEAD2, which yields fewer redundant messages than those generated by S1PD and SEAD1. This fact amounts to the applied conditions for message selection. Indeed, contrary to SEAD1, SEAD2 discard any redundant message regardless the side it came from.

From another side, the adaptation of the SEAD protocol versions performance with relation to the deployed application requirements is ensured through the configurable parameter called $\alpha$. The effectiveness of such parameter is evaluated through Fig. 6(a) and Fig. 6(b). In Fig. 6(a), we illustrate the impact of $\alpha$ parameter on the redundancy ratio, for a vehicles' density equal to 65 vehicles $/ \mathrm{km}$. As we can notice, two basic parts of the curve can be distinguished: an increasing part for $\alpha$ value ranging from 1.5 to 2.8 , and from 1.5 to 2 , for SEAD1 and SEAD2, respectively. Then a steady state is achieved from a certain value of $\alpha$. The increasing part generally indicates the configurable phase of the proposed protocol. Fig. 6(b), clearly confirm the impact of $\alpha$ values, ranging from 1.5 to 2 , on the achieved PDR. Thus, on the basis of the plotted curve we can clearly fix the accurate value of $\alpha$ responsible for maintaining a particular amount of data reachability.

Furthermore, we observe, that the $\alpha$ configuration range determined within the analytic model is still valid in realistic scenario. In particular, we notice through Fig. 6(b) that the analytic values of PDR fit the data reachability performance achieved by SEAD2 protocol, under realistic environment, although our model corresponds to SEAD1. This may be explained by the fact that the PDR model is function of the number of hops which is clearly affected by the nodes' mobility factor (that generates different number of vehicles in each segment). Concerning the radio model, we have integrated the realistic radio model in Equation (23) by conditioning the number of vehicles receiving a packet in each segment in accordance with this radio model. A difference of only $2 \%$ in the $\mathrm{PDR}$ is noticed in comparison with the perfect radio range results and consequently it is not shown in the figure. Thus, we can confirm that the protocol configuration is not sensitive to the propagation model.

As the main focus of VANET applications is ensuring the passenger's safety and reducing the loss of life, we propose in the next section to study the efficiency of SEAD1 and SEAD2 toward safety message dissemination. To this end, we tune the alpha value to 2 for the rest of simulations.

b) The Protocols Scalability Feature: After determining the effective values of $\alpha$, we next move to configure SEAD versions in a way to be adapted for safety applications. The aim of this section is to evaluate the protocols performance in scalable network.

At first glance, we can notice, through Fig. 7(b), that both SEAD versions significantly succeed in reducing the number of transmissions. Indeed, the number of forwarders drastically degrades with SEAD1 and SEAD2, about 50\% in dense network compared to S1PD. Moreover, we can perceive that the forwarding ratio decreases when the vehicles' density increases. This behavior shows the effect of node selection strategy on reducing unnecessary transmissions. As a result, SEAD1 and SEAD2 are efficient in terms of reducing the number of packet transmission hops, which has a great impact on the broadcast latency, since less relaying nodes are involved in the re-forwarding process.

However, a specific focus on SEAD versions, allows us to notice that SEAD2 presents slightly lower rate of forwarders than SEAD1. This result is expected due to the message reforwarding condition adopted in each version. Contrary to SEAD2, SEAD1 does not discard all redundant messages. It considers that each redundant message coming from upstream could be useful to enhance network reliability. As receiving the same message from upstream does not mean that it has 


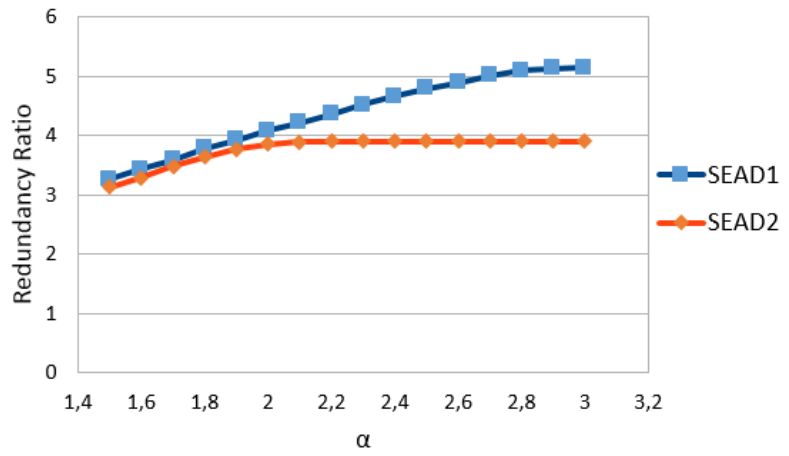

(a) Redundancy Ratio

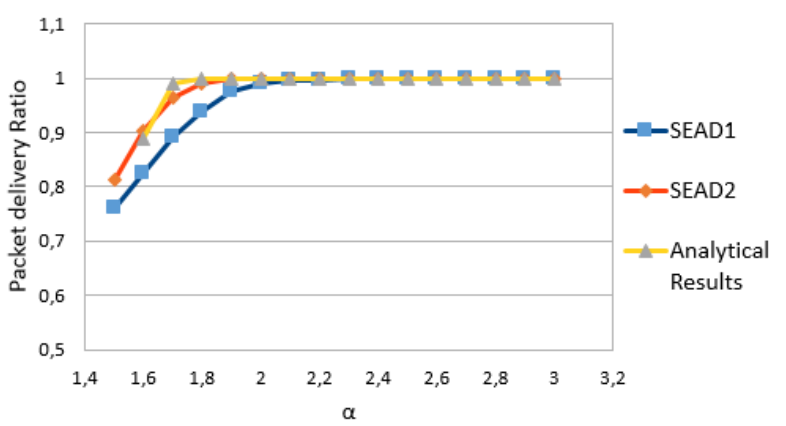

(b) Packet Delivery Ratio

Figure 6: Protocols performance behavior under various $\alpha$ value

reached vehicles further in the message direction. This message should be then retransmitted.

On the other hand, we observe, through Fig. 7(c) that SEAD1 and SEAD2 are able to practically achieve the same packet delivery ratio, as it is reached by S1PD, while decreasing the number of forwarding vehicles. In other words, both of SEAD versions are able to guarantee a high data reachability while saving the network capacity and the limited bandwidth consumption.

This observation is further proved through Fig. 7(d) from which we compare the amount of received bits per unit of time using both SEAD versions with that received under S1PD scheme. Indeed, both SEAD schemes outperform S1PD in terms of "link load", especially for high dense network, which illustrates the protocol scalability. This result amounts to the adaptivity side of the proposed protocols by which a substantial decline in the proportion of redundant transmissions is achieved. As Fig. 7(a) shows, the redundancy ratio presented by both SEAD versions, exhibits a threshold effect, according to which " $\mathrm{r}$ " value remains constant (does not exceed 4 from a vehicles' density equal to $60 \mathrm{veh} / \mathrm{km}$ ) while the vehicles' density is increasing. This enhancement, achieved through an efficient use of the limited bandwidth, leads to save the network capacity and offer the possibility for different applications to be simulta- neously run.

In addition, the efficiency of network resources consumption, using SEAD1 and SEAD2, is further shown via the drop ratio metric plotted in Fig. 7(e). A significant decline in the proportion of erroneous received messages has almost reached $80 \%$, compared to that presented with S1PD. Such result, may clearly show the inherent effect of both SEAD versions on enhancing the data dissemination reliability by reducing non-successful message reception. Dealing with safety applications, SEAD1 and SEAD2 protocols have shown high efficiency to mitigate the broadcast storm problem by decreasing the network contention and collisions compared to S1PD. Since the transmission delay is a crucial metric for safety message dissemination, SEAD1 and SEAD2, have shown the best performance in terms of end-to-end delay compared to S1PD, as presented in Fig. 7(f). In particular, SEAD2 is capable to reduce up to $50 \%$ the transmission delay, while ensuring a total data reachability for the entire network. This result is explained through the fact that, due to SEAD1 design more redundant transmissions are introduced, which allows to more collisions between forwarders within the last segment. As a result, forwarders in the following segment will take the relay for data forwarding at the cost of more transmission delay. 


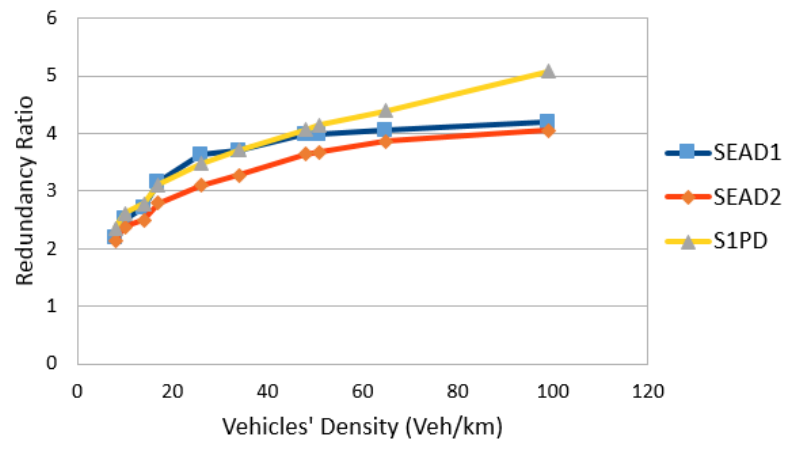

(a) Redundancy Ratio

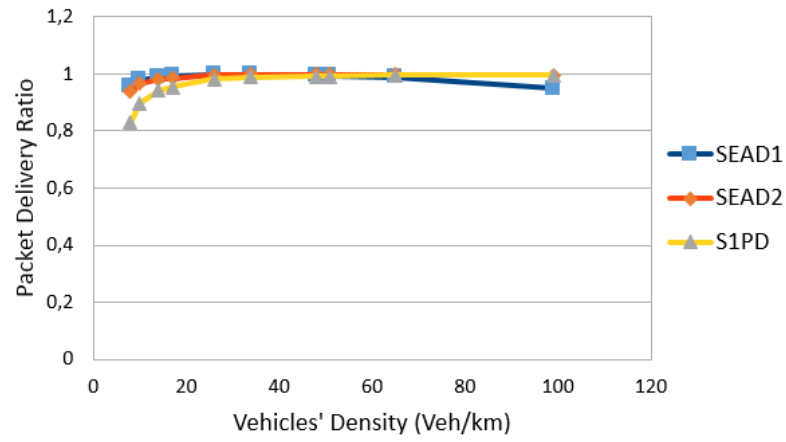

(c) Packet Delivery Ratio

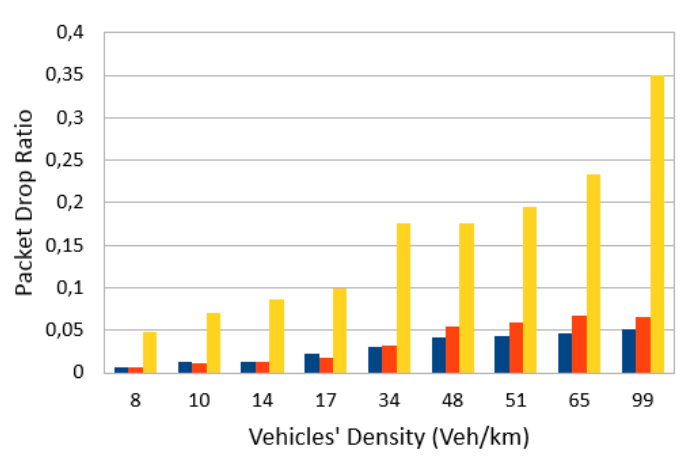

(e) Packet Drop Ratio

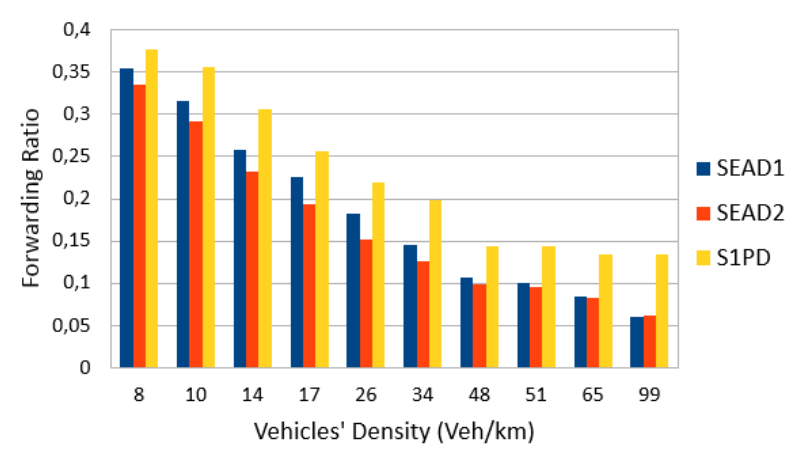

(b) Forwarding Ratio

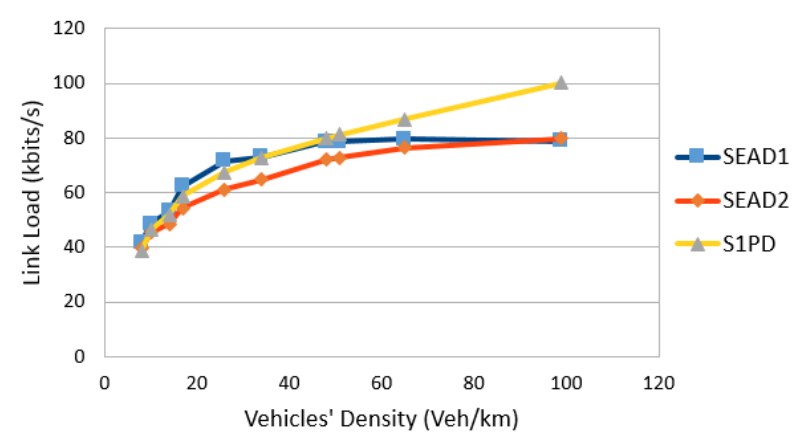

(d) Link Load

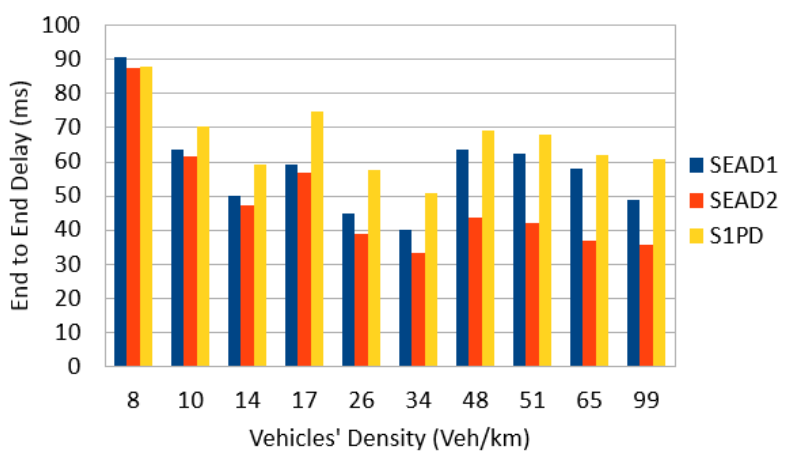

(f) End to End Delay

Figure 7: Protocols performances behavior under Various Vehicles' Density 
In order to thoroughly compare the different considered protocols, we propose in Fig. 8 a radar chart of 4 metrics that could be practically combined together, which are the Forwarding Ratio, the End-to-End delay, the Link Load and the Packet drop ratio. The performance evaluation of the considered protocols is conducted under a high dense Network (99 vehicles/km) in order to evaluate their scalability feature. Based on the Radar chart, we can easily deduce the efficiency of SEAD protocol compared to S1PD. In particular, both SEAD1 and SEAD2 have significantly reduced the number of forwarders participating in the dissemination process which has conducted to a significant decrease in the dropped packets. This result may explain the decrease of the Link Load and End-to-End delay since less number of transmissions is needed in order to achieve a high packet delivery ratio (Fig. 7(c)). Furthermore, we can deduce that SEAD2 slightly outperforms SEAD1 since it presents less transmission delay.

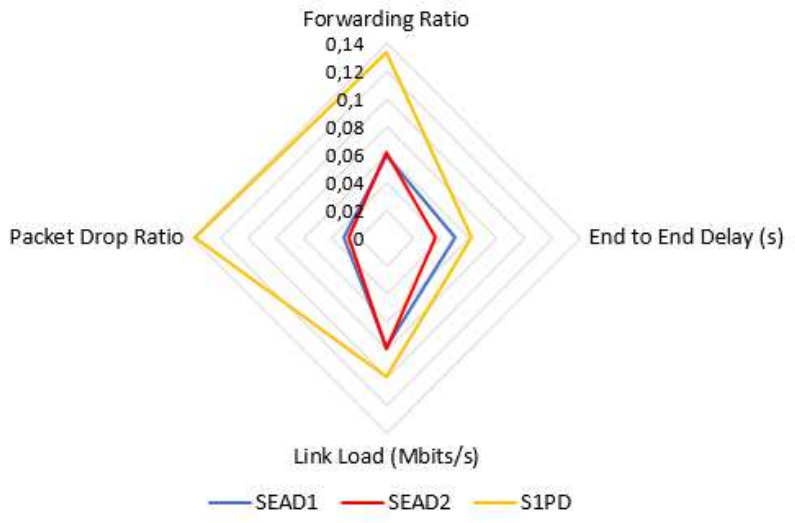

Figure 8: Protocols' performance evaluation under a high dense network

Assuming these salient properties, it is worthy to confirm the protocols (SEAD1 and SEAD2) efficiency toward real time applications, especially, safety-related applications.

\section{Concluding remarks and fu- ture works}

In this work, we presented an analytic model that aims to study the data dissemination performance in vehicular environment. In particular, this model attempts to investigate the mean redundancy ratio behavior during the dissemination process and its impact on the data reachability. The studied protocols in this work are largely inspired from a simple and efficient data dissemination protocol denoted "SEAD". The purpose behind these protocols (SEAD1 and SEAD2) is to mitigate the broadcast storm effect in a scalable vehicular network. Thus, an efficient broadcast strategy is designed to take into account different network parameters (transmitter-receiver distance, message direction, message redundancy) without need for beacon messages exchange. The originality of these protocols, is that they can be effectively adapted to fit different application types under different performance requirements. Via an " $\alpha$ " parameter we are able to adjust the amount of redundancy necessary to guarantee a particular level of packet delivery ratio.

Through the proposed model, we proved that the protocols efficiency is achieved under a proper configuration of the redundancy parameters. Our findings showed that the protocols setting is possible within a specific range of $\alpha$ values. Moreover, we obtained explicit values for $\alpha$ able to guarantee a certain amount of PDR with a minimum ratio of network resources consumption. Think that makes the protocols configuration more practical and easier to be integrated in real VANET system (OBU).

Given the accurate value of $\alpha$, we tuned the protocols performance to fully fit safety-related applications. We carried out several simulations to confirm the protocols efficiency in realistic environment by achieving high data reachability within a low transmission delay while optimizing the limited network resources consumption. Moreover, a particular comparison between the two protocols leads to deduce that SEAD2 slightly outperforms SEAD1 in terms of resources consumption and end-to-end delay. This result is expected in non-perfect propagation model 
where the transmission range is variable.

In future work, we plan to study the protocols performance in sparse network and enhance their design to mitigate the intermittent connectivity problem. In addition, we intend to further reduce the network resources consumption in the presence of different application types through the network coding scheme, taking into consideration its networking complexity.

This work is a part of the MOBIDOC project achieved under the PASRI program, funded by the European Union and administrated by the ANPR.

\section{References}

[1] Achour, I., Bejaoui, T., Busson, A., Tabbane, S.: Sead: A simple and efficient adaptive data dissemination protocol in vehicular ad-hoc networks. Wireless Networks pp. 1-11 (2015)

[2] Achour, I., Bejaoui, T., Busson, A., Tabbane, S.: Delay-based strategy for safety message dissemination in vehicular ad hoc networks: Slotted or continuous? In: Wireless Communications and Mobile Computing Conference (IWCMC), 2016 International, pp. 268-274. IEEE (2016)

[3] Baiocchi, A., Salvo, P., Cuomo, F., Rubin, I.: Understanding spurious message forwarding in vanet beaconless dissemination protocols: An analytical approach. IEEE Transactions on Vehicular Technology 65(4), 2243-2258 (2016)

[4] Basheer, H.S., Bassil, C.: A review of broadcasting safety data in v2v: Weaknesses and requirements. Ad Hoc Networks 65, 13-25 (2017)

[5] Chaqfeh, M., Lakas, A., Jawhar, I.: A survey on data dissemination in vehicular ad hoc networks. Vehicular Communications 1(4), 214-225 (2014)

[6] DRIVE-SAFELY.NET: http://www.drivesafely.net/safe-following-distance/

[7] ETSI, E.: 637-3: Its. Vehicular Communications; Basic Set of Applications; Part 2: Specification of Decentralized Environmental Notification Basic Service (2014)
[8] ETSI, T.: Intelligent transport systems (its); vehicular communications; basic set of applications; definitions. Tech. rep., Tech. Rep. ETSI TR 102638 (2009)

[9] Fracchia, R., Meo, M.: Analysis and design of warning delivery service in intervehicular networks. Mobile Computing, IEEE Transactions on $7(7), 832-845$ (2008)

[10] Group, I..W., et al.: Ieee standard for information technology-telecommunications and information exchange between systemslocal and metropolitan area networks-specific requirements-part 11: Wireless lan medium access control (mac) and physical layer (phy) specifications amendment 6: Wireless access in vehicular environments. IEEE Std 802, 11p (2010)

[11] Hafi, H., Abdou, W., Merniz, S.: Adaptivesegmentation and flexible-delay based broadcasting protocol for vanets. In: International Conference on Wired/Wireless Internet Communication, pp. 207-218. Springer (2017)

[12] Khabazian, M., Aissa, S., Mehmet-Ali, M.: Performance modeling of message dissemination in vehicular ad hoc networks with priority. Selected Areas in Communications, IEEE Journal on 29(1), 61-71 (2011)

[13] Khabazian, M., Aïssa, S., Mehmet-Ali, M.: Performance modeling of safety messages broadcast in vehicular ad hoc networks. Intelligent Transportation Systems, IEEE Transactions on 14(1), 380-387 (2013)

[14] Kumar, R., Dave, M., et al.: A review of various vanet data dissemination protocols. International Journal of U-and E-Service, Science and Technology (IJUNESST) 5(3), 27-44 (2012)

[15] Liu, C., Chigan, C.: Rpb-md: Providing robust message dissemination for vehicular ad hoc networks. Ad Hoc Networks 10(3), 497-511 (2012)

[16] Mirani, F., Busson, A., Adjih, C.: Improving delay-based data dissemination protocol in 
vanets with network coding. REV Journal on Electronics and Communications 2(3-4) (2013)

[17] NS3: NS3 - Network Simulator project. https://www.nsnam.org/

[18] Panichpapiboon, S., Ferrari, G.: Irresponsible forwarding. In: ITS Telecommunications, 2008. ITST 2008. 8th International Conference on, pp. 311-316. IEEE (2008)

[19] Panichpapiboon, S., Pattara-Atikom, W.: A review of information dissemination protocols for vehicular ad hoc networks. Communications Surveys \& Tutorials, IEEE 14(3), 784-798 (2012)

[20] Resta, G., Santi, P., Simon, J.: Analysis of multi-hop emergency message propagation in vehicular ad hoc networks. In: Proceedings of the 8th ACM international symposium on Mobile ad hoc networking and computing, pp. 140-149. ACM (2007)

[21] Rostami, F., Wan, T.C.: E1pd: Enhanced 1persistence data dissemination protocol for vehicle ad hoc networks in highway environments. In: Telecommunication Technologies (ISTT), 2012 International Symposium on, pp. 137-141. IEEE (2012)

[22] Schwartz, R.S., Barbosa, R.R., Meratnia, N., Heijenk, G., Scholten, H.: A directional data dissemination protocol for vehicular environments. Computer Communications 34(17), 2057-2071 (2011)

[23] Schwartz, R.S., Das, K., Scholten, H., Havinga, P.: Exploiting beacons for scalable broadcast data dissemination in vanets. In: Proceedings of the ninth ACM international workshop on Vehicular inter-networking, systems, and applications, pp. 53-62. ACM (2012)

[24] Schwartz, R.S., Scholten, H., Havinga, P.: A scalable data dissemination protocol for both highway and urban vehicular environments. EURASIP Journal on Wireless Communications and Networking 2013(1), 1-19 (2013)
[25] Segata, M., Dressler, F., Cigno, R.L.: Jerk beaconing: A dynamic approach to platooning. In: Vehicular Networking Conference (VNC), 2015 IEEE, pp. 135-142. IEEE (2015)

[26] SUMO: Sumo - simulation of urban mobility. http://sumo.sourceforge.net/

[27] Tseng, Y.C., Ni, S.Y., Chen, Y.S., Sheu, J.P.: The broadcast storm problem in a mobile ad hoc network. Wireless networks 8(2-3), 153-167 (2002)

[28] Vinel, A., Koucheryavy, Y., Andreev, S., Staehle, D.: Estimation of a successful beacon reception probability in vehicular ad-hoc networks. In: Proceedings of the 2009 International Conference on Wireless Communications and Mobile Computing: Connecting the World Wirelessly, pp. 416-420. ACM (2009)

[29] Vinel, A.V., Dudin, A.N., Andreev, S.D., Xia, F.: Performance modeling methodology of emergency dissemination algorithms for vehicular adhoc networks. In: CSNDSP, pp. 397-400 (2010)

[30] Wisitpongphan, N., Tonguz, O.K., Parikh, J.S., Mudalige, P., Bai, F., Sadekar, V.: Broadcast storm mitigation techniques in vehicular ad hoc networks. Wireless Communications, IEEE 14(6), 84-94 (2007) 